Research Paper

\title{
Identification of a Prognostic Signature Model with Tumor Microenvironment for predicting Disease-free Survival after Radical Prostatectomy
}

\author{
Hao Zhao ${ }^{{ }^{*}}$, Xuening Zhang ${ }^{1}$, Zhan Shi ${ }^{2}$, Bingxin Guo ${ }^{3}$, Wenli Zhang ${ }^{1}$, Kun He${ }^{1}$, Xueqi Hu${ }^{1}$, Songhe Shi ${ }^{\circledR}$ \\ 1. Department of Epidemiology and Biostatistics, College of Public Health, Zhengzhou University, Zhengzhou 450001, China. \\ 2. Department of Medicine, Zhengzhou First People's Hospital, Zhengzhou 450004, China. \\ 3. Department of Urology, Henan Province Hospital of Traditional Chinese Medicine, Zhengzhou 450002, China \\ *? \\ $\square$ Corresponding author: Prof Songhe Shi, Department of Epidemiology and Biostatistics, College of Public Health, Zhengzhou University, No.100 Science \\ Avenue, Zhengzhou, 450001, People's Republic of China. E-mail: ssh@zzu.edu.cn, Tel: 86-18037689587.
}

() The author(s). This is an open access article distributed under the terms of the Creative Commons Attribution License (https://creativecommons.org/licenses/by/4.0/). See http:/ /ivyspring.com/terms for full terms and conditions.

Received: 2020.07.27; Accepted: 2021.01.18; Published: 2021.02.22

\begin{abstract}
Background: The tumor microenvironment (TME) and immune checkpoint inhibitors have been shown to promote active immune responses through different mechanisms. We attempted to identify the important prognostic genes and prognostic characteristics related to TME in prostate cancer (PCa).

Methods: The gene transcriptome profiles and clinical information of PCa patients were obtained from The Cancer Genome Atlas (TCGA) database, and the immune and stromal scores were calculated by the ESTIMATE algorithm. We evaluated the prognostic value of the risk score (RS) model based on univariate Cox analysis and least absolute shrinkage and selection operation (LASSO) Cox regression analysis and established a nomogram to predict disease-free survival (DFS) in PCa patients. The GSE70768 dataset was utilized for external validation. Twenty-two subsets of tumor-infiltrating immune cells were analyzed using the CIBERSORT algorithm.

Results: In this study, the patients with higher immune/stromal scores were associated with a worse DFS, higher Gleason score, and higher pathological T stage. Based on the immune and stromal scores, 515 differentially expressed genes (DEGs) were identified. The univariate Cox and LASSO Cox regression models were employed to select 18 DEGs from 515 DEGs and construct an RS model. The DFS of the high-RS group was significantly lower than that of the low-RS group $(P<0.001)$. The AUCs for the 1-year, 3-year and 5-year DFS rates in the RS model were $0.890,0.877$ and 0.841 , respectively. A nomogram of DFS was established based on the RS and Gleason score, and the AUCs for the 1-year, 3-year and 5-year DFS rates in the nomogram were $0.907,0.893$, and 0.872 , respectively. These results were further validated in the GSE70768 dataset. In addition, the proportion of Tregs was determined to be higher in high-RS patients $(P<0.05)$, and the expression levels of five immune checkpoints (CTLA-4, PD-1, LAG-3, TIM-3 and TIGIT) were observed to be higher in high-RS patients $(P<0.05)$.

Conclusions: Our study established and validated an 18-gene prognostic signature model associated with TME, which might serve as a prognosis stratification tool to predict DFS in PCa patients after radical prostatectomy.
\end{abstract}

Key words: prostate cancer; radical prostatectomy; microenvironment; stromal; immune; prognosis; prediction

\section{Introduction}

Prostate cancer (PCa) is the most common malignant tumor among men in Western world [1]. According to the 2018 Global Cancer Report, the incidence of prostate cancer among men is second only to lung cancer, and the incidence is gradually increasing, with an annual growth rate as high as $8.92 \%[2,3]$. With the progress of cancer treatment technology, localized PCa can be cured by radical prostatectomy (RP), and mortality is also significantly reduced. However, the recurrence rate of PCa after RP 
is still high, resulting in treatment failure. Previous studies have reported that approximately $40 \%$ of patients relapse within five years after $\mathrm{RP}$, and approximately $27-53 \%$ of patients eventually develop local recurrence or distant metastasis within 10 years after RP $[4,5]$.

In recent years, immunotherapy with cytokines and immune checkpoint inhibitors has been shown to promote active immune responses through different mechanisms [6]. Therefore, a new classification of $\mathrm{PCa}$ in combination with immunotherapy is needed to more accurately predict postoperative recurrence, thereby contributing to clinical decision making to achieve personalized treatment and reduce the recurrence rate among PCa patients.

The tumor microenvironment (TME) refers to the surrounding microenvironment of tumor cells, including immune cells, stromal cells, endothelial cells, inflammatory cells, and fibroblasts [7]. Among these cells, tumor-infiltrating immune cells and stromal cells are two major non-tumor cell components, which have been considered important for the diagnosis and prognostic evaluation of cancer patients [8]. Therefore, understanding the cell composition and function of the TME has considerable potential to effective prevent cancer recurrence and promote immunotherapy responses.

Bioinformatics analysis uses a combination of biological, statistical, computer science, and informatics techniques to process and analyze large amounts of complex biological data [9]. The establishment of public databases, such as The Cancer Genome Atlas (TCGA) database and Gene Expression Omnibus (GEO) database, provides new data resources and technical means for TME research [10, 11]. Yoshihara et al [12] first proposed the ESTIMATE (Estimation of Stromal and Immune cells in Malignant Tumor tissues using Expression data) algorithm in 2013. This algorithm uses the unique properties of the transcription profile of cancer samples to infer infiltrating stromal/immune cells. According to reports, researchers have explored the tumor characteristics and prognostic assessment of lung cancer [13], breast cancer [14], and clear-cell renal cell carcinoma [15] based on the ESTIMATE algorithm. However, the value of immune and stromal scores for PCa has not been verified to data.

In this study, we examined the TME in PCa patients, calculated the immune and stromal scores for each cancer sample, and established a risk score (RS) prognostic model and a nomogram combining the RS and Gleason score using the TCGA database. Moreover, these results were validated using the GEO database. Finally, based on the CIBERSORT (Cell type Identification by Estimating Relative Subpopulations of RNA Transcription) method, we explored the relationship between high-RS and low-RS PCa patients and immune cell infiltration and immune checkpoints to provide a foundation for future efforts to achieve precise immunotherapy and postoperative management of PCa patients.

\section{Materials and Methods}

\section{Data collection and processing}

We obtained the fragments per kilobase million (FPKM) data of RNA-Seq from the TCGA-PRAD cohort (https://portal.gdc.cancer.gov/), including 499 PCa patients and 52 normal samples. Next, the FPKM data was transferred to transcripts per million (TPM) expression data. The gene expression levels of duplicate samples were averaged, and normal samples were deleted for subsequent analysis.

We used the Genomic Data Commons (GDC) tool and cBiopPortal website (http://www. cbioportal.org/) to download the corresponding clinical information, including age, pathological $\mathrm{T}$ stage, lymph node status, Gleason score, surgical margin status, tumor laterality, and prognostic information. We excluded samples with incomplete key clinical information, and finally included $480 \mathrm{PCa}$ patients for the following analysis. We utilized the "limma" package for normalization processing. Next, immune, stromal and ESTIMATE scores were calculated using the ESTIMATE algorithm. For GEO database, the inclusion criteria were as follows: (1) patients diagnosed with PCa; (2) patients who had undergone RP; and (3) patients with detected gene levels in tissue samples. The exclusion criteria were as follows: (1) clinical data without prognostic information and (2) dataset with a small sample size $(n<50)$. Finally, the eligible dataset, GSE70768 $(n=111)$, was selected, and the normalized expression matrix was used for subsequent analysis.

\section{Correlation analysis and survival analysis}

For data satisfying the parameter tests, the $t$-test was utilized for comparisons between two groups, and ANOVA was employed for comparisons of three groups or more. For test data with the unsatisfactory parameters, the Wilcoxon test was used for comparisons between two groups, and the Kruskal-Wallis analysis was used for comparisons of three groups or more. The relationship between the immune/stromal score and important clinical phenotypes was explored by comparing the differences in the immune/stromal score in different clinical subgroups. Disease-free survival (DFS), as the main prognostic endpoint, was defined as the time from the date of diagnosis to the date of recurrence or death and the last follow-up. 
According to the stromal/immune score of each PCa patient, the best cut-off value based on the R package "maxstat" (i.e., the maximum selective rank statistic method) was used to divide the patients into high and low score groups [16]. Based on "survival" packages, the difference in DFS between the two groups was evaluated by the Kaplan-Meier (K-M) method and log-rank test.

\section{Differentially expressed gene (DEG) screening}

The "limma" package in R software was used to screen for DEGs between the high and low groups of immune/stromal scores. In this study, an adjusted $P$ value $<0.05$ and a fold change $\geq 1.5$ were regarded as the critical values for screening DEGs. The immune-related DEGs and stromal-related DEGs showing the same expression trend were selected using a Venn diagram. We used the "ggplot2" and "pheatmap" packages to generate a volcano plot and heatmap.

\section{DEG functional enrichment analysis}

The David online database (http:/ / david.ncifcrf. gov) was used to explore the potential functions of DEGs. Gene ontology (GO) analysis included biological processes (BPs), molecular functions (MFs) and cellular components (CCs), which were demonstrated by bar plots. The Kyoto Encyclopedia of Genes and Genomes (KEGG) was used to conduct the pathway analysis, which was illustrated by a dotplot. With a false discovery rate (FDR) $<0.05$ as the cut-off value, all enrichment results were visualized with the "ggplot2" package.

\section{Establishment of a prognostic signature model and survival analysis}

A univariate Cox model was used to determine the relationship between TME-related DEG expression and DFS. Next, least absolute shrinkage and selection operator (LASSO) Cox regression analysis was used to develop an optimal risk signature with the minimum number of genes [17]. A set of genes and their coefficients were determined by the minimum criteria, which involved selecting the best penalty parameter $\lambda$ associated with the 10 -fold cross validation [18]. The RS was calculated as follows: $\mathrm{RS}=\sum\left(\beta \mathrm{i}{ }^{*}\right.$ Expi) $\left(' \mathrm{i}^{\prime}=\right.$ the number of prognostic hub genes, ' $\beta i^{\prime}$ represents the coefficient of each gene, and 'Expi' represents gene expression.) In addition, PCa patients were divided into high-RS and low-RS groups according to the optimal cut-off value of the risk score. A receiver operating characteristic (ROC) curve was then used to assess the predictive ability of the RS model. The K-M method and log-rank test were used to analyze the differences in survival between the high-RS group and the low-RS group.

Validation of the prognostic signature model in the test dataset

The GSE70768 independent dataset was used for verification. A scatter plot was used to show the distribution of gene expression profiles and the RS, and the Pearson correlation coefficient was used calculate the correlation. Immunohistochemistry (IHC) images of the selected prognosis-related genes in high- and low-grade tissue were retrieved from the Human Protein Atlas online database (http://www. proteinatlas.org). According to the RS calculation formula of the training dataset, the samples in the test dataset were divided into the high-RS group and the low-RS group. K-M survival analysis and ROC curve analysis were used to evaluate the predictive ability of this model.

\section{Identification of independent prognostic factors}

Univariate and multivariate Cox analysis were used to study the independent prognostic value of the RS and other clinical characteristics, and a clinical prediction model was established using a nomogram. Next, the performance of the nomogram was evaluated by time-dependent ROC curve analysis.

\section{Estimating the composition of immune cells}

CIBERSORT is a deconvolution algorithm based on the principle of linear support vector regression used to describe the infiltration of immune cells in the sample. LM22 is composed of 547 genes that accurately distinguish 22 human hematopoietic cell phenotypes, including seven $\mathrm{T}$ cell types, naïve and memory B cells, plasma cells, NK cells, and myeloid subsets [19]. We utilized CIBERSORT and LM22 to jointly estimate the scores of 22 human immune cell types in PCa samples from the TCGA cohort. For each sample, the sum of all estimated immune cell type scores was equal to 1 . We compared differences in the composition of immune cell types between high-RS and low-RS groups.

\section{Statistical analysis}

Statistical analysis was performed using $\mathrm{R}$ software (version 3.6.1). All statistical tests were two-sided, and a $P$ value $<0.05$ was considered to be significant.

\section{Results}

Immune score and stromal score were correlated with clinical features of $\mathrm{PCa}$

The workflow chart of this study is shown in Figure S1. A total of 480 male PCa patients were 
included in the TCGA database. Elderly PCa patients ( $\geq 65$ years) accounted for $33.54 \%$. There were 186 patients $(38.75 \%)$ with $\leq$ pT2c stage disease, 153 patients $(31.88 \%)$ with pT3a stage disease, and 141 patients $(29.38 \%)$ with $\geq$ pT3b stage disease. Regarding the Gleason score, 44 cases $(9.17 \%)$ were in the $<7$ score group, 240 cases $(50 \%)$ were in the 7 score group, and 196 cases $(40.83 \%)$ were in the $>7$ score group. For other detailed information, see Table 1 for clinical information.

The immune, stromal and ESTIMATE scores of each sample were calculated using an ESTIMATE algorithm. The immune score ranged from -1404.50 to 2963.33, the stromal score ranged from -1897.04 to 1762.53, and the ESTIMATE score ranged from -3237.41 to 3584.35 . The relationship between immune and stromal scores and clinical characteristics showed that a higher immune score was significantly associated with a higher $\mathrm{T}$ stage $(P=0.015)$, positive surgical marginal status $(P=0.038)$ and lymph node metastasis $(P=0.047)$ (Figure 1A). A higher stromal score was significantly associated with a higher $\mathrm{T}$ stage $(P<0.001)$ and higher Gleason score $(P=0.002)$ (Figure 1B). The relationship between the ESTIMATE score and clinical characteristics was similar to that of the stromal score (Figure S2).

\section{Immune score and stromal score were significantly related to $\mathrm{PCa}$ prognosis}

The K-M survival curves of the relationship between immune and stromal scores and PCa patient prognosis showed that patients with lower immune and stromal scores had higher DFS rates $(P=0.011$ and $P=0.037$, respectively) (Figure $1 \mathrm{D}$ and 1E). PCa patients with low ESTIMATE scores also consistently had higher DFS rates than patients with high ESTIMATE scores $(P=0.02)$ (Figure 1F). These observations consistently suggested that patients with a low immune or stromal score had a more favorable outcome.

\section{Identification of DEGs based on the immune score and stromal score in PCa}

To explore the DEGs that are closely related to the TME, the "limma" package was used to process the Affymetrix microarray data from $480 \mathrm{PCa}$ patients. Figure 2A shows a heatmap of 804 DEGs between high and low immune scores, and Figure 2B shows a heatmap of 1098 DEGs between high and low stromal scores.

In addition, the volcano plot shows DEGs based on the immune score and stromal score (Figure S3). For the immune score, there were 68 up-regulated DEGs and 736 down-regulated DEGs in the high group compared with the low group. For stromal score, compared with the low score group, there were 104 up-regulated DEGs and 994 down-regulated DEGs in the high score group. A Venn diagram showed 41 cross-up-regulated DEGs and 474 cross-down-regulated DEGs between the immune and stromal groups (Figure 2C).

\section{Function and pathway enrichment analysis of DEGs}

Functional enrichment analysis for DEGs, including BPs, CCs, MFs and KEGG pathways were conducted using the David gene annotation tool. BPs indicated that these genes may be associated with the immune response, inflammatory response, cell adhesion, extracellular matrix organization, and leukocyte migration. CCs indicated that these genes may be associated with the plasma membrane and extracellular exosome, region, and space. MFs indicated that these genes may be associated with calcium ion binding, receptor activity, and serine-type endopeptidase activity. The results of KEGG enrichment were related to the immune response, including phagosomes, infection, cytokine receptor interactions, and cell inflammatory molecules (CAMs) (Figure 2D). Overall, our results confirmed that TME-related DEGs were closely related to the anti-tumor immunity of PCa patients.

\section{Establishment of a prognostic signature model and survival analysis}

To explore the potential role of DEGs in DFS, a univariate Cox proportional hazards regression model was first developed, and the results showed that 172 prognostic genes were selected by univariate analysis. Next, the 10-fold cross-validation random sampling method was used, and according to the -2 log-likelihood test, through repeated calculation and verification, the model was optimized at the penalty parameter $\log \lambda=-1.59$, and a risk score (RS) model of 18 genes was constructed (Figure 3A, 3B). RS $=\left(0.06410^{*} \mathrm{C} 1 \mathrm{QC}\right)+\left(0.00467^{*} \mathrm{COL} 1 \mathrm{~A} 1\right)+\left(0.01777^{*}\right.$ HOPX $)+(0.17512 *$ ITGAX $)+(0.35768 *$ STAB1 $)+$ $\left(0.13676^{*} \mathrm{TGFB} 1\right)+\left(-0.04815^{*} \mathrm{APOF}\right)+\left(-0.06180^{*}\right.$ CHRNA2) + $\left(-0.22487^{*}\right.$ CLIC6 $)+(-0.03191 *$ EGR1 $)+$ $(-0.01361 * \mathrm{FEV})+\left(-0.00269^{*} \mathrm{FOS}\right)+\left(-0.06470^{*} \mathrm{GJB} 1\right)+$ $(-0.23936 *$ GNG2 $)+(-0.10059 *$ HSD11B1 $)+\left(-0.03021^{*}\right.$ OLFML3 $)+(-0.06134 *$ PLTP $)+(-0.03940 *$ TGM3 $)$ (Figure S4). In addition, survival curves of 18 DEGs were constructed to explore the prognostic value of each gene (Figures S5 and S6). These results of K-M curves showed that $\mathrm{PCa}$ patients with low expression levels of C1QC, COL1A1, HOPX, ITGAX, STAB1, and TGFB1 had a better prognosis. In contrast, we found that low expression of other hub genes was associated with a poor prognosis in PCa patients. 
A

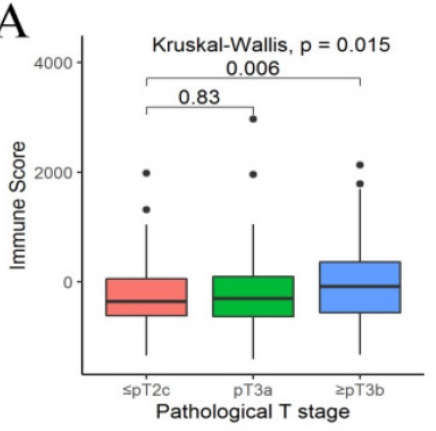

B

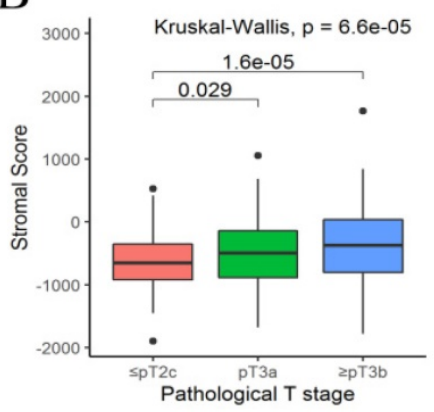

C
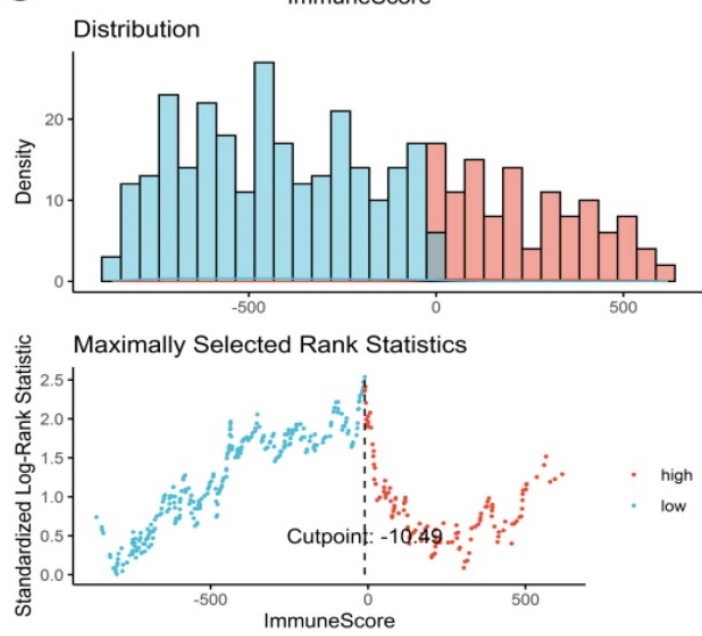

E

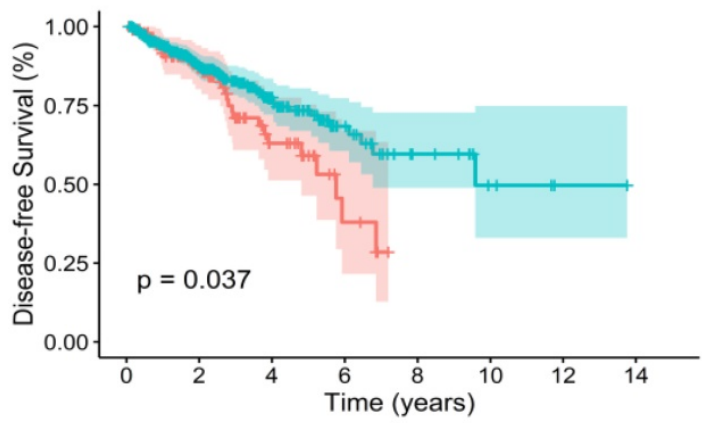

Number at risk

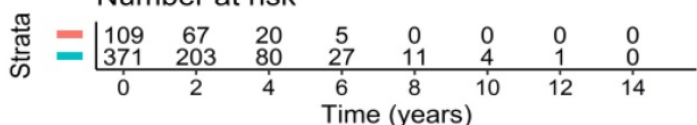

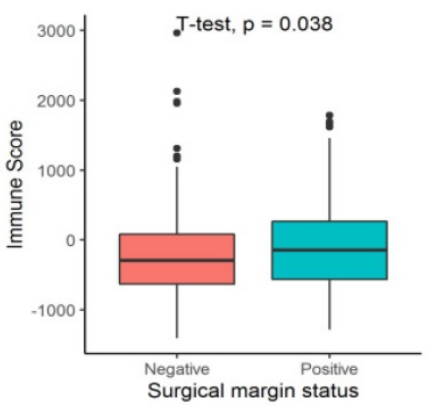

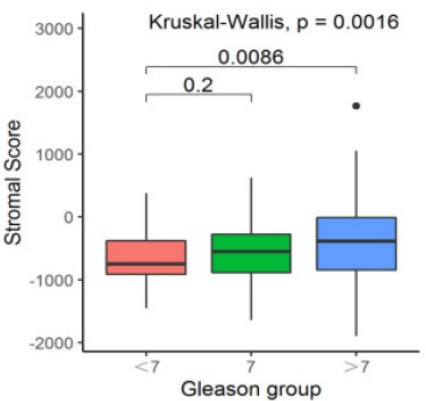

D
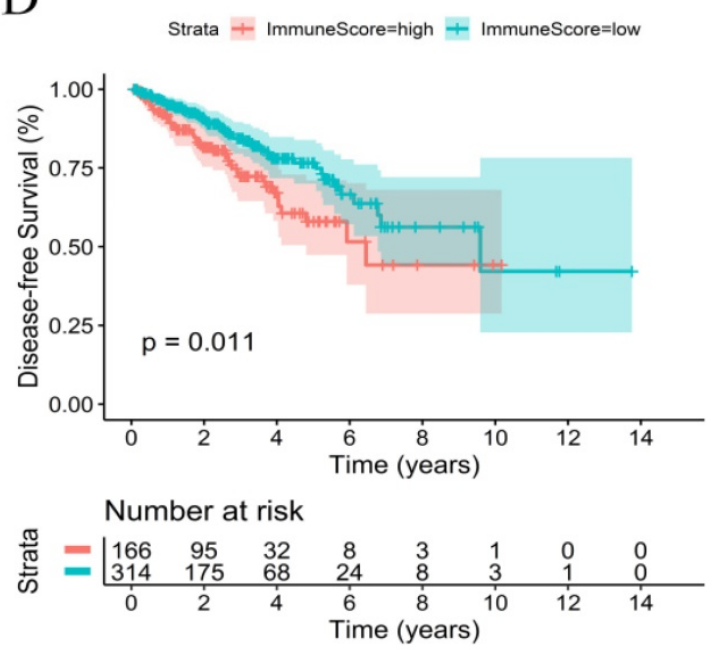

F

Strata + ESTIMATEScore=high + ESTIMATEScore=low

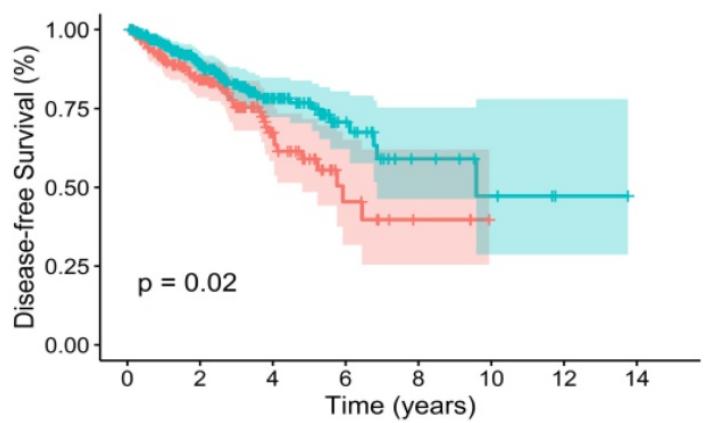

Number at risk

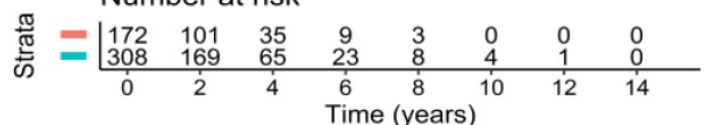

Figure 1. Immune score and stromal score were correlated with clinical features and prognosis of PCa. (A) Immune score correlated with clinical features. (B) Stromal score correlated with clinical features. (C) The best cut-off value for the immune score. (D-F) K-M survival curves of the relationship between the immune, stromal and ESTIMATE scores and PCa patient prognosis. 
A

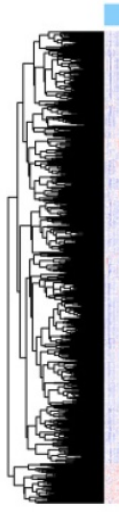

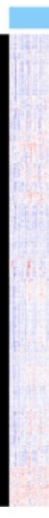

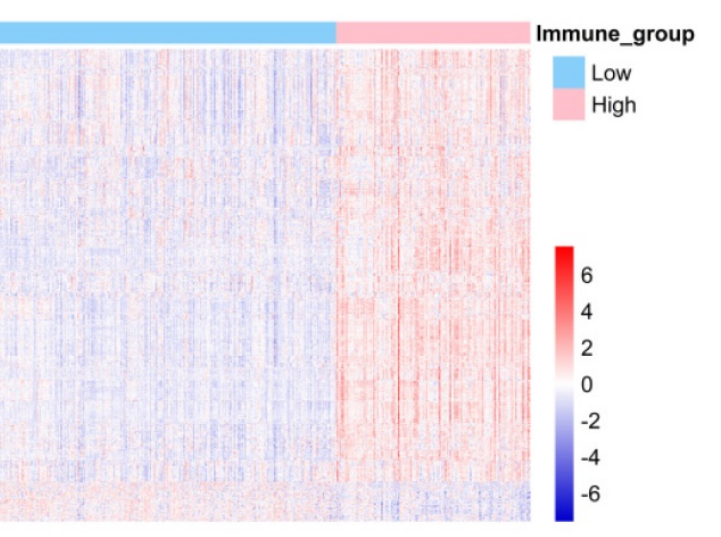

B

D
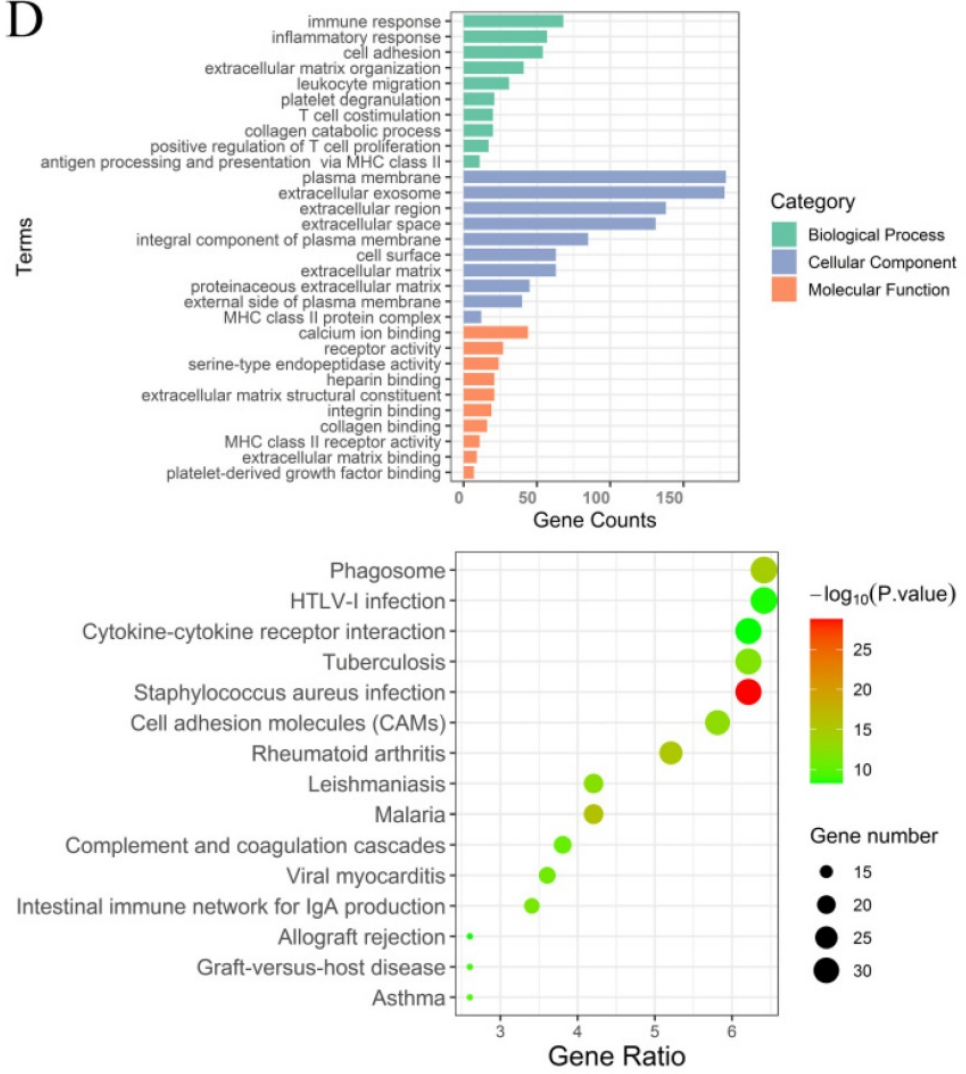

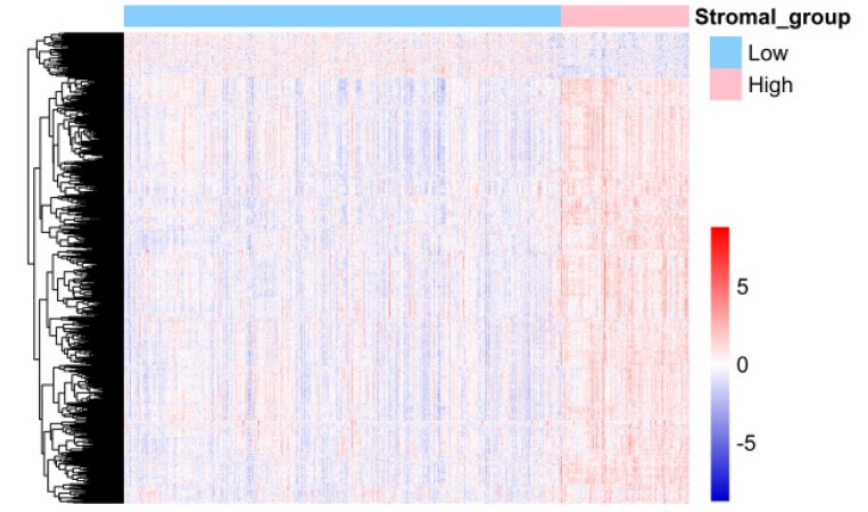

Stromal group

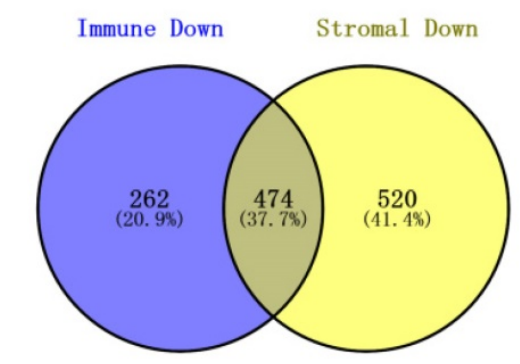

C

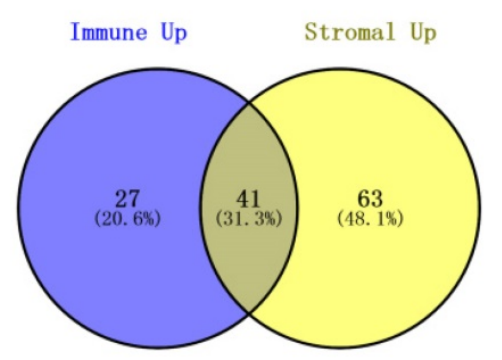

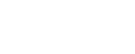


in the Gleason score model were 0.704, 0.677 and 0.682, respectively (Figure S7C), and we found that the RS model was more accurate for predicting

A

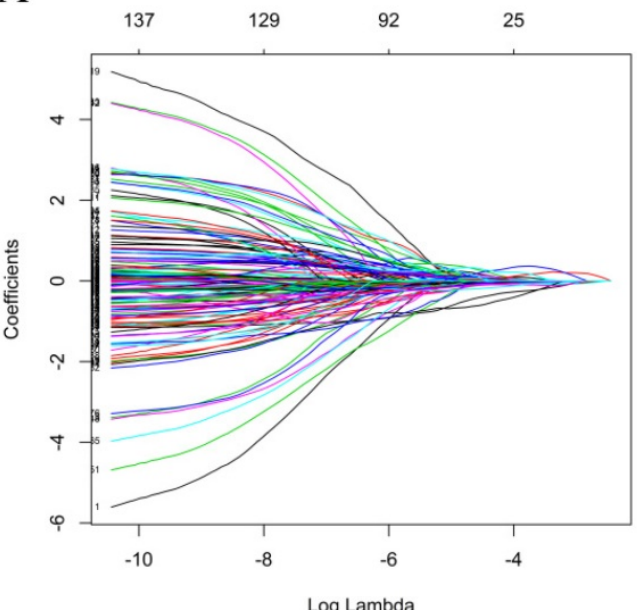

C

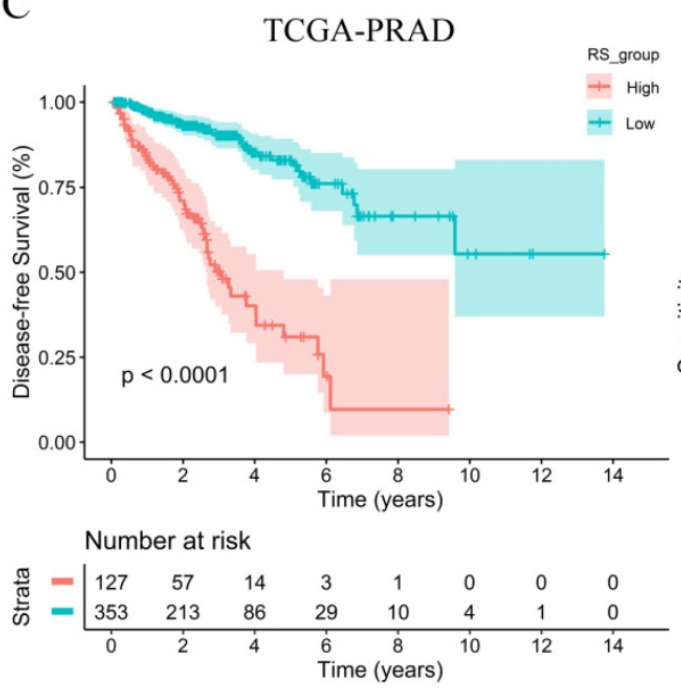

E

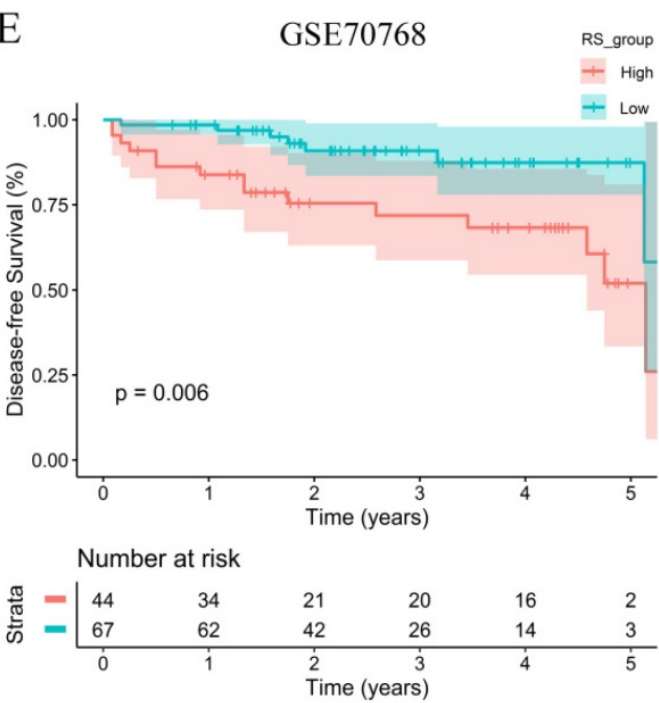

prognosis than the Gleason score model in the TCGA database.

B $\begin{array}{llllllllllll}137 & 134 & 133 & 129 & 119 & 95 & 81 & 54 & 31 & 20 & 9 & 3\end{array}$

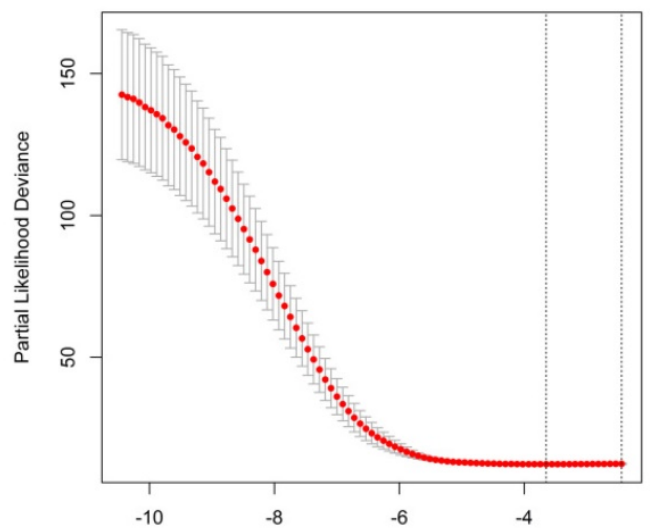

D

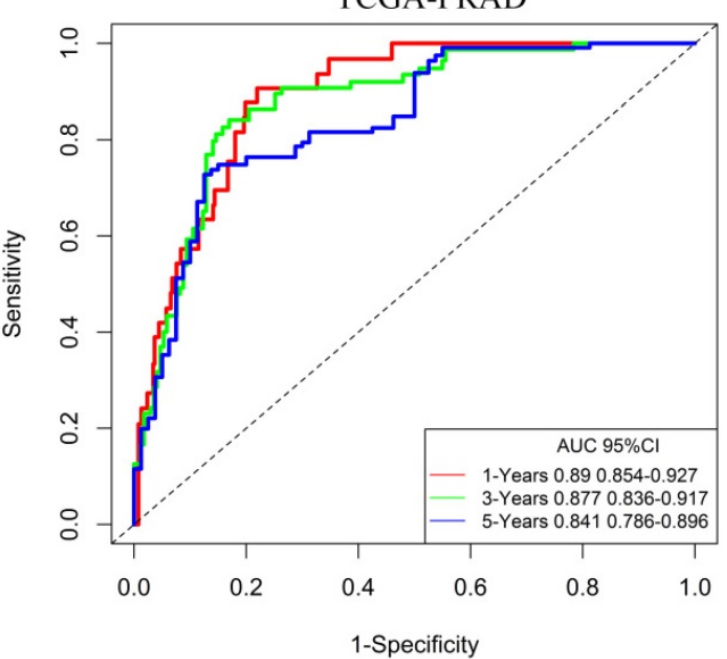

F

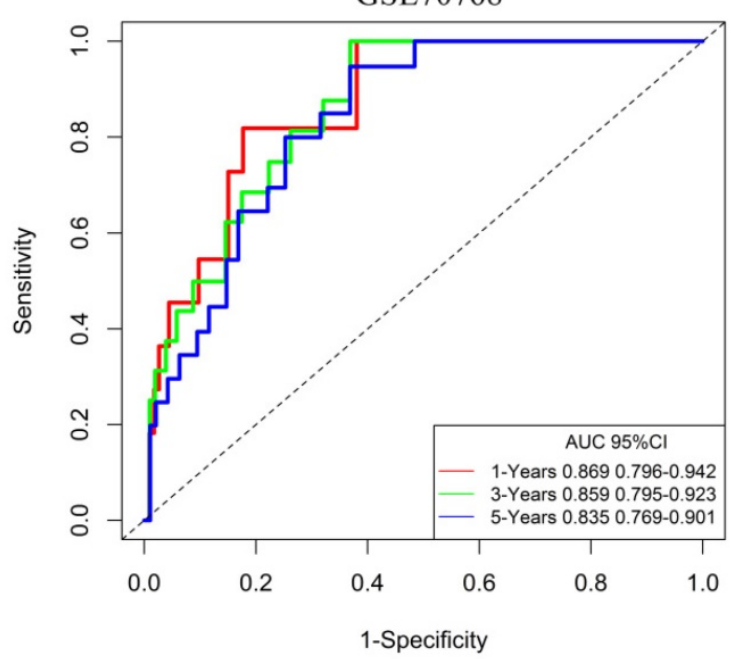

Figure 3. RS model calculation and survival analysis. (A) LASSO coefficient profiles. (B) The 10-fold cross-validation results that identified optimal values of the penalty parameter $\lambda$. (C) K-M curves of the high- and low-RS groups in the TCGA-PRAD dataset. (D) ROC curve based on the RS model in the TCGA-PRAD dataset. (E) K-M curves of the high- and low-RS groups in the GSE70768 dataset. (F) ROC curve based on the RS model in the GSE70768 dataset. 

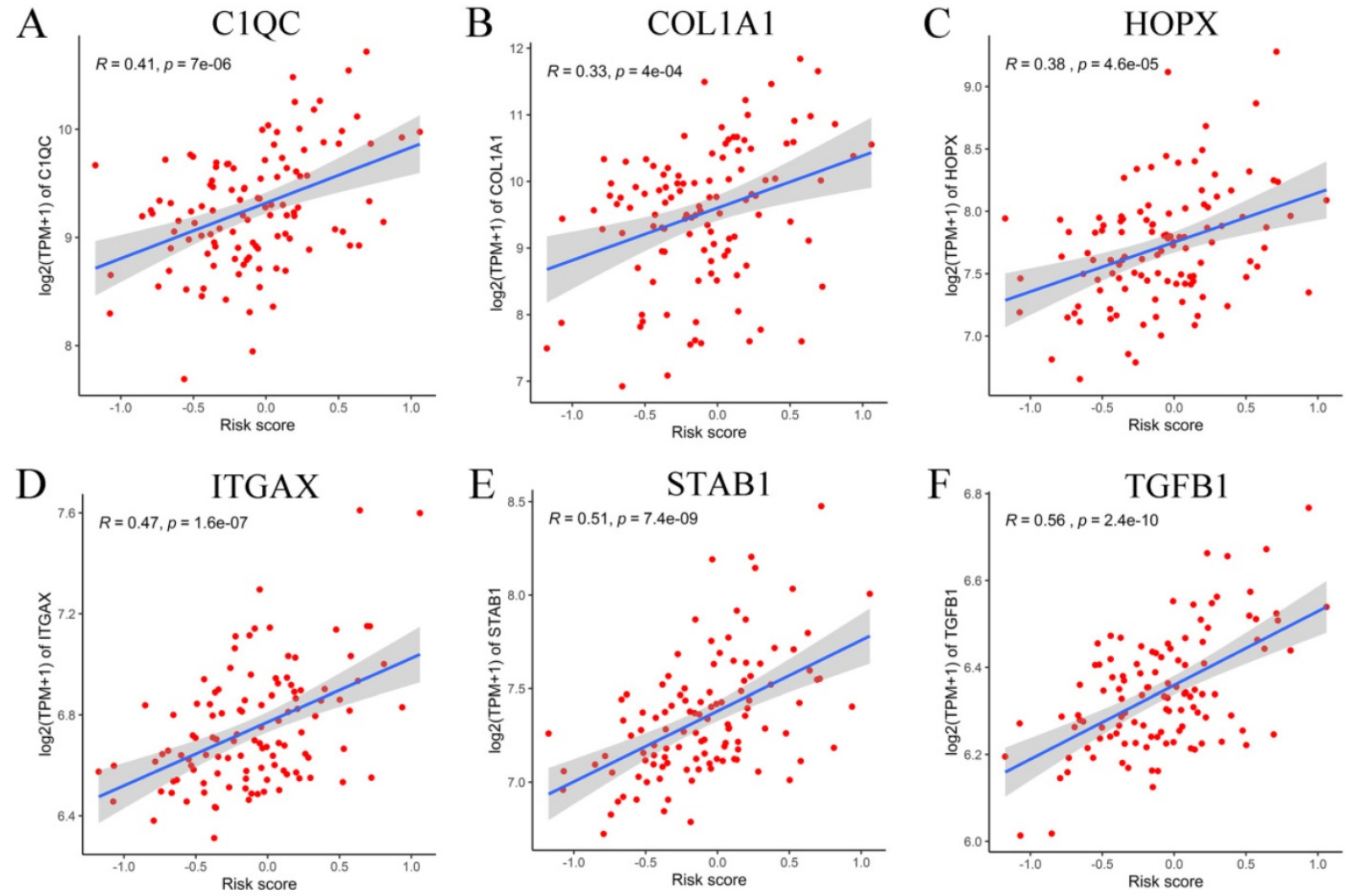

Figure 4. Correlation between six adverse genes and RS in GEO database. (A-F) The correlation between six genes and the RS indicated that C1QC, COLIAI, HOPX, ITGAX, STABI, and TGFB1 were positively correlated with the RS.

Table 1. Clinical characteristics of $480 \mathrm{PCa}$ patients included in the study from the TCGA cohort

\begin{tabular}{|c|c|c|c|c|}
\hline \multirow[t]{2}{*}{ Variables } & \multirow{2}{*}{$\begin{array}{l}\text { Whole cohort } \\
(\mathrm{N}=480)\end{array}$} & \multicolumn{2}{|c|}{ Relapse or death } & \multirow[t]{2}{*}{ Log-rank $P$} \\
\hline & & No $(N=386)$ & Yes $(N=94)$ & \\
\hline \multicolumn{4}{|l|}{ Age } & \multirow[t]{3}{*}{0.462} \\
\hline$<65$ & $319(66.46)$ & $257(80.56)$ & $62(19.44)$ & \\
\hline$\geq 65$ & $161(33.54)$ & $129(80.12)$ & $32(19.88)$ & \\
\hline \multicolumn{4}{|c|}{ Tumor laterality } & \multirow[t]{3}{*}{0.212} \\
\hline Left/Right & $56(11.67)$ & $48(85.71)$ & $8(14.29)$ & \\
\hline Bilateral & $424(88.33)$ & 338 (79.72) & $86(20.28)$ & \\
\hline \multicolumn{4}{|c|}{ Pathological T stage } & \multirow[t]{4}{*}{$<0.001$} \\
\hline$\leq \mathrm{pT} 2 \mathrm{c}$ & $186(38.75)$ & $170(91.40)$ & $16(8.60)$ & \\
\hline рТ3а & $153(31.88)$ & $120(78.43)$ & $33(21.57)$ & \\
\hline$\geq \mathrm{pT} 3 \mathrm{~b}$ & $141(29.38)$ & $96(68.09)$ & 45 (31.91) & \\
\hline \multicolumn{4}{|c|}{ Lymph node status } & \multirow[t]{3}{*}{0.007} \\
\hline $\mathrm{pN} 0 / \mathrm{pNx}$ & $402(83.75)$ & $331(82.34)$ & $71(17.66)$ & \\
\hline pN1 & 78 (16.25) & 55 (70.51) & $23(29.49)$ & \\
\hline \multicolumn{4}{|c|}{ Gleason score } & \multirow[t]{4}{*}{$<0.001$} \\
\hline$<7$ & 44 (9.17) & 43 (97.73) & $1(2.27)$ & \\
\hline 7 & $240(50.00)$ & 214 (89.17) & $26(10.83)$ & \\
\hline$>7$ & $196(40.83)$ & $129(65.82)$ & $67(34.18)$ & \\
\hline \multicolumn{4}{|c|}{ Surgical margin status } & \multirow[t]{3}{*}{$<0.001$} \\
\hline Negative & $333(69.38)$ & $283(84.98)$ & $50(15.02)$ & \\
\hline Positive & 147 (30.63) & $103(70.07)$ & $44(29.93)$ & \\
\hline
\end{tabular}

\section{Validation of the prognostic signature model}

To verify the generalization value of the RS model based on the TCGA cohort, we calculated the risk score of each sample for the 111 PCa patients in the GSE70768 cohort using the above mentioned RS formula. The K-M survival curve indicated that the low-RS group had a higher DFS $(P=0.006)$ (Figure 3E). In addition, the ROC curves based on the RS model showed that the AUCs for the 1-year, 3-year and 5 -year DFS rates were 0.869, 0.859 and 0.835, respectively (Figure 3F). The ROC curves based on the Gleason score model showed that the AUCs for the 1-year, 3-year and 5-year DFS rates were 0.724, 0.695 and 0.688 , respectively (Figure S7D). Therefore, the results of the validation set also indicated that the RS model had better robustness and was superior to the Gleason score model. In addition, the correlation between 18 genes and the RS indicated that C1QC, COL1A1, HOPX, ITGAX, STAB1, and TGFB1 were positively correlated with the RS (Figure 4), while the others were negatively correlated (Figure S8). The human protein atlas database was used to explore protein expression levels. Typical IHC of six adverse and eight favorable prognostic genes (except APOF, CHRNA2, FEV, and OLFML3, which are not included in the database) in high grade and low grade PCa tissues are shown in Figure 5 and Figure S9, respectively.

\section{Identification of independent prognostic factors}

The univariate Cox model analysis showed that a higher pathological $\mathrm{T}$ stage, Gleason score and RS, 
lymph node metastasis, and positive surgical margin status were risk factors affecting prognosis. In the multivariate Cox analysis, the meaningful variables from the univariate Cox model analysis were included, and the results showed that after the adjustment for variables including variables such as the lymph node status, pathological T stage, Gleason score, and surgical margin status, the RS was independent predictor (HR: 4.04, 95\% CI: 0.25-2.44), similar to and independent of the Gleason score (Table 2).

The nomogram of the predicted DFS was further established based on the RS and Gleason score (Figure 6A). The AUCs for the 1-year, 3-year and 5-year DFS rates of the nomogram were 0.9070 .893 and 0.872 , respectively (Figure 6B). Further validation of an independent cohort of $111 \mathrm{PCa}$ patients in the GSE70768 cohort also showed good predictive power (Figure 6C).

A

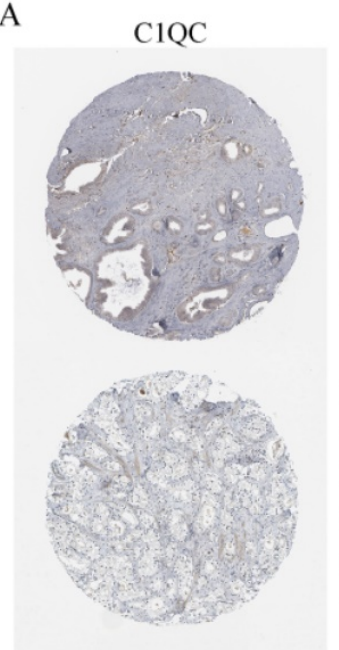

D

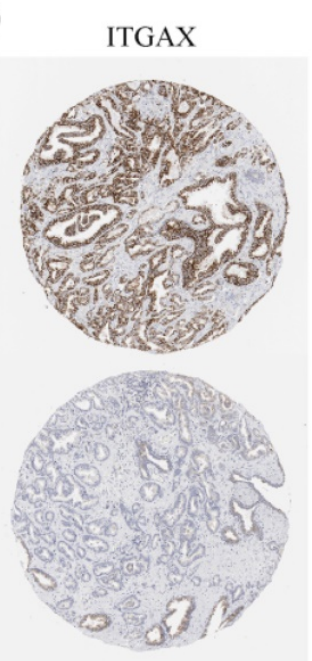

High grade
Male, age 71
Patient id: 640
Staining: Low
Intensity: Weak
Quantity: $>75 \%$
Low grade
Male, age 58
Patient id: 3302
Staining: Not detected
Intensity: Negative
Quantity: None

rand

High grade

Male, age 73

Patient id: 3301

Staining: High

Intensity: Strong

Quantity: $>75 \%$

Low grade

Male, age 59

Patient id: 1052

Staining: Medium

Intensity: Moderate

Quantity: $75 \%-25 \%$

E
B
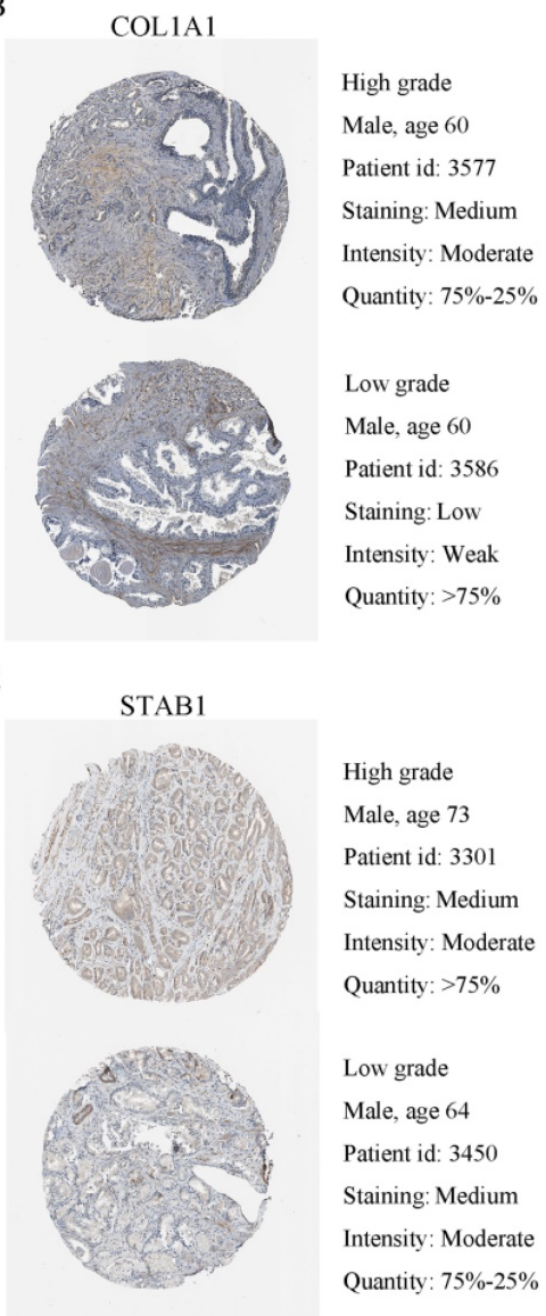

Estimating the composition of immune cells

We used CIBERSORT to estimate the immune cell composition of 480 samples and to quantify the relative levels of different cell types in the mixed cell population (Figure S10). As shown in Figure 7, we compared different cell types of patients in the low-RS group with those in the high-RS group. These results indicated that the expression levels of resting memory CD $4 \mathrm{~T}$ cells, CD $8 \mathrm{~T}$ cells, M1 macrophages and eosinophils in the low-risk group were significantly higher than those in the high-risk group $(P<0.05)$. In contrast, the expression levels of M2 macrophages, regulatory T cells (Tregs) and dendritic cells resting in the high-RS group were significantly higher than those in the low-RS group $(P<0.05)$.

We also explored the expression of immune checkpoints between the high-RS and low-RS PCa patients. The expression levels of CTLA-4, PD-1, LAG-3, TIM-3 and TIGIT in high-RS patients were significantly higher than those in low-RS patients $(P<0.05)$ (Figure 8).

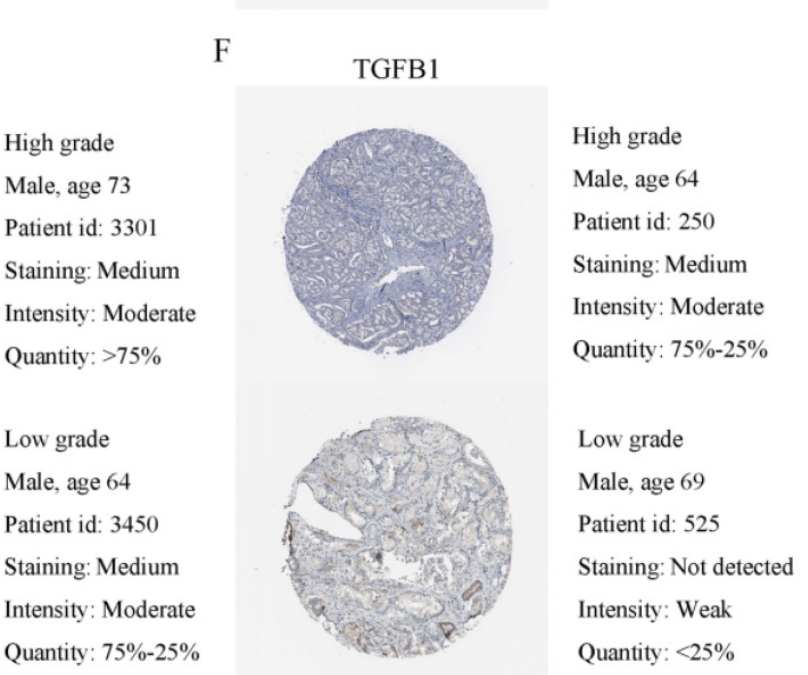

Figure 5. Human protein atlas database was used to explore six adverse expression levels. (A-F) Typical IHC of six adverse prognostic genes in high grade and low grade PCa tissues. 
A
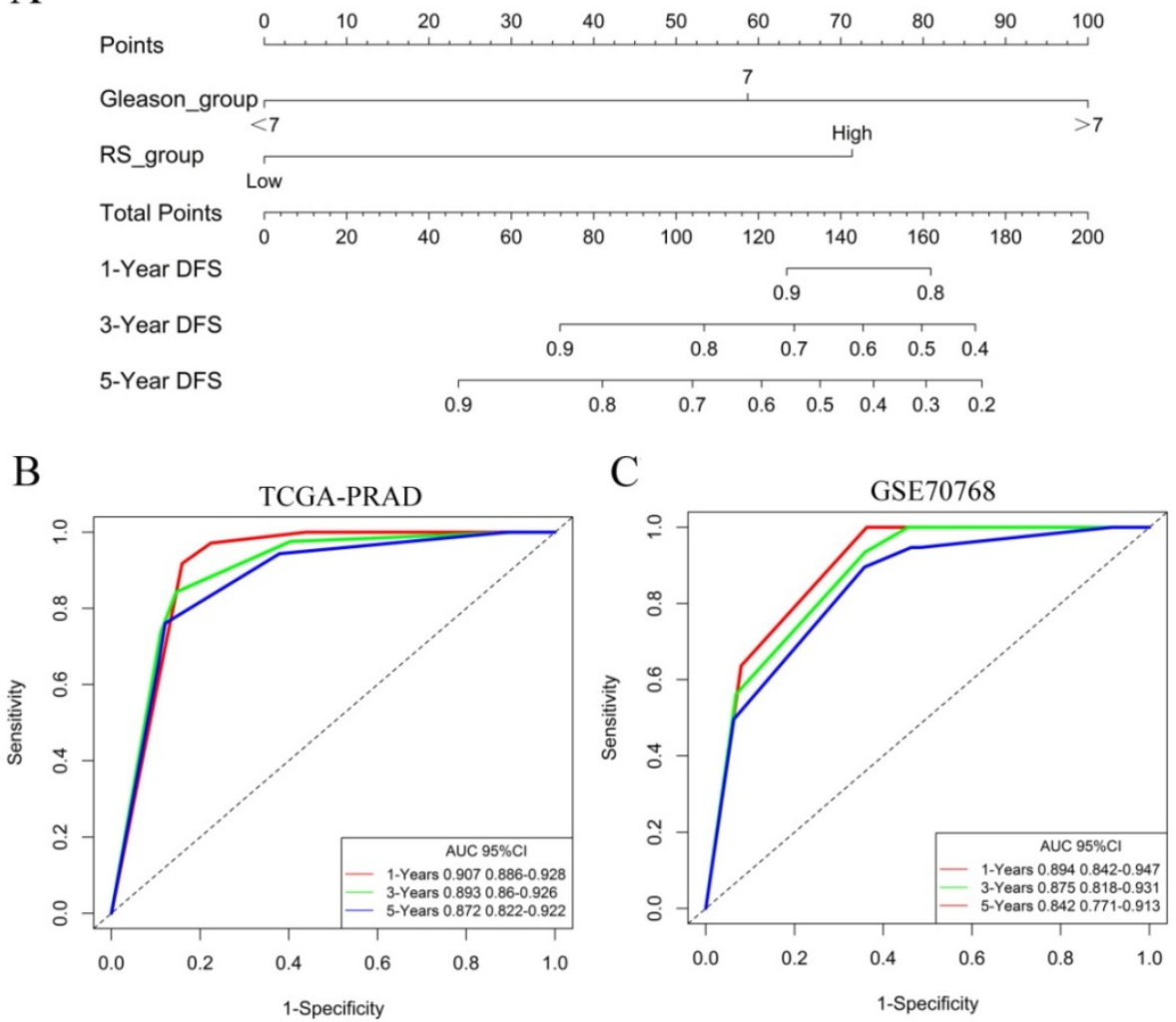

Figure 6. Identification of independent prognostic factors. (A) The nomogram for predicting DFS was established based on the RS and Gleason score. (B) The AUCs for the 1-year, 3-year and 5-year DFS rates of the nomogram in the TCGA-PRAD dataset. (C) The AUCs for the 1-year, 3-year and 5-year DFS rates of the nomogram in the GSE70768 dataset.

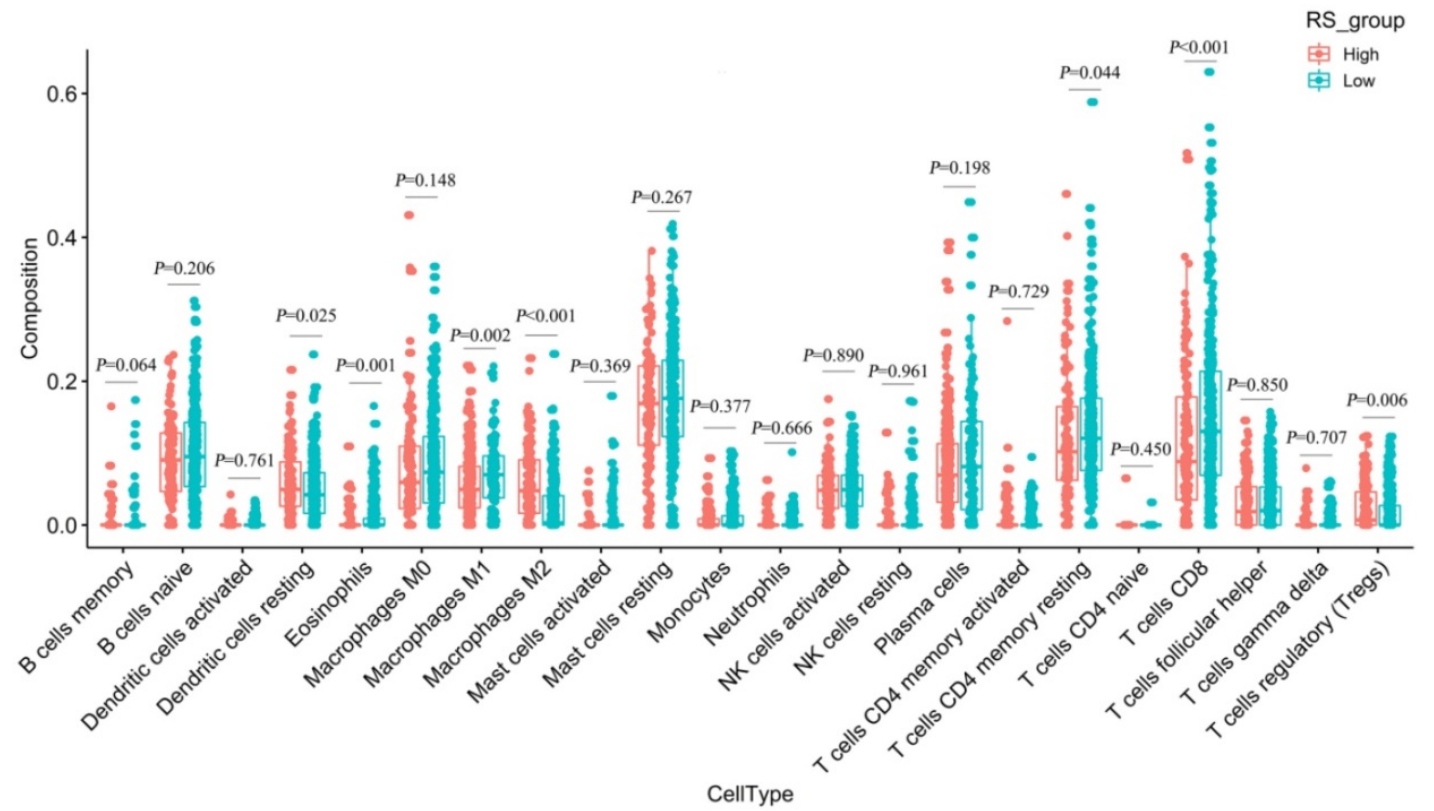

Figure 7. Differences in 22 immune cells between the high-RS and low-RS groups.

\section{Discussion}

Previous studies have shown that the TME plays a vital role in the development, progression and recurrence of cancer [20, 21]. However, due to the heterogeneity and complexity of the TME, only some
PCa patients benefit from immunotherapy. It remains critical to construct an effective model for accurately predicting the prognosis of PCa patients. To the best of our knowledge, this study is the first to apply the ESTIMATE algorithm to identify a TME-related prognostic signature of PCa patients after RP. 
A

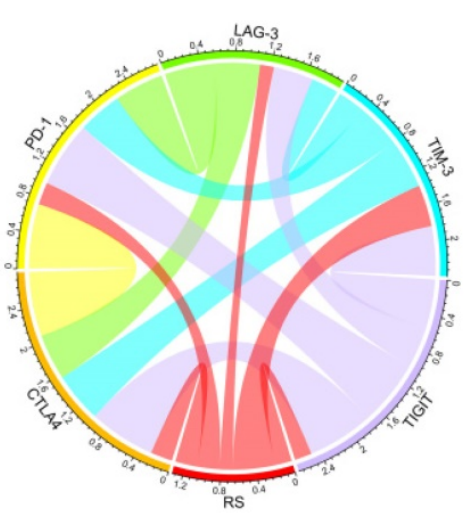

D

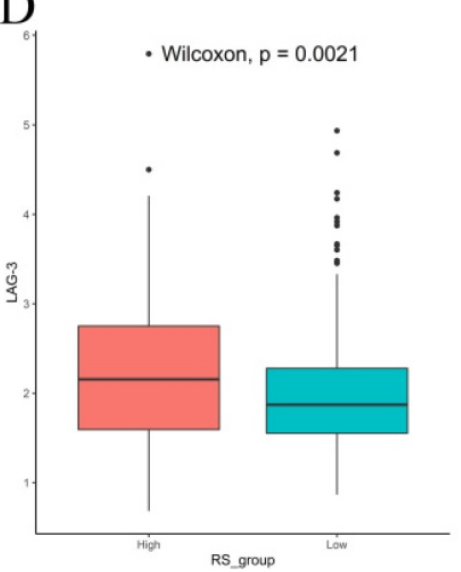

B

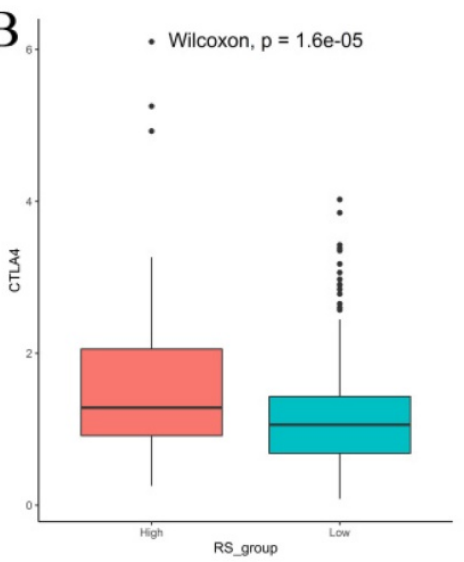

E

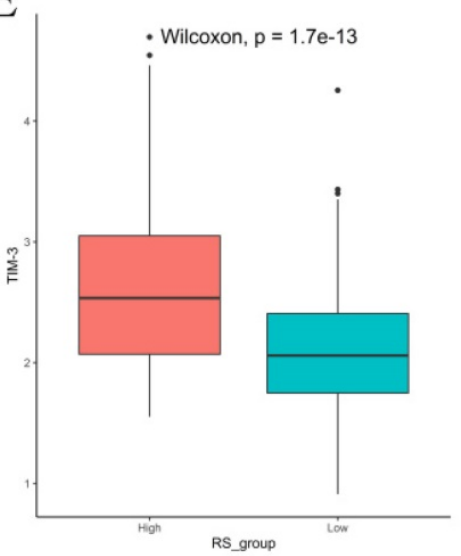

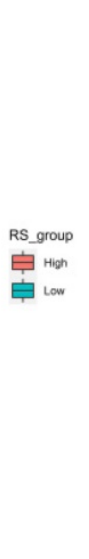

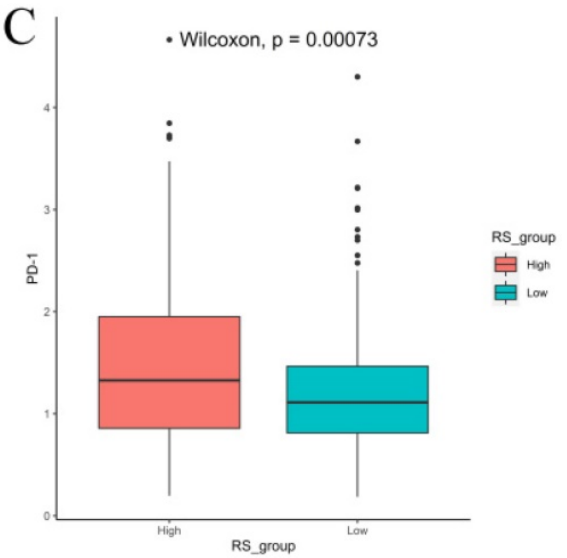

$\mathrm{F}$

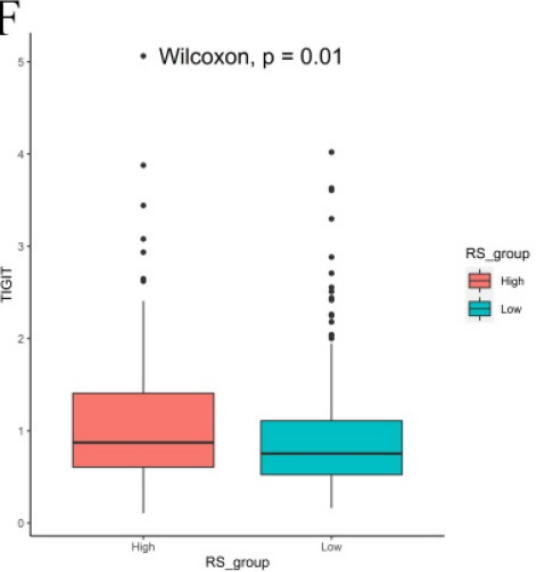

Figure 8. Expression of immune checkpoints between high-RS and low-RS PCa patients. (A) Correlation of the RS with the expression levels of several prominent immune checkpoints. (B-F) Box figure showing significantly different immune checkpoints between high-RS and low-RS patients.

Table 2. Univariate and multivariate Cox analysis of clinical information and the RS

\begin{tabular}{|c|c|c|c|c|}
\hline \multirow[t]{2}{*}{ Variables } & \multicolumn{2}{|c|}{ Univariate analysis } & \multicolumn{2}{|c|}{ Multivariate analysis } \\
\hline & $\mathrm{HR}(95 \% \mathrm{CI})$ & $P$ & HR $(95 \% \mathrm{CI})$ & $P$ \\
\hline \multicolumn{5}{|l|}{ Age } \\
\hline$<65$ & 1 & & & \\
\hline$\geq 65$ & $0.46(0.77-1.8)$ & 0.462 & & \\
\hline \multicolumn{5}{|c|}{ Tumor laterality } \\
\hline Left/Right & 1 & & & \\
\hline Bilateral & $1.58(0.77-3.27)$ & 0.215 & & \\
\hline \multicolumn{5}{|c|}{ Pathological T stage } \\
\hline$\leq \mathrm{pT} 2 \mathrm{c}$ & 1 & & 1 & \\
\hline pT3a & $1.8(1.43-4.77)$ & 0.002 & $1.49(0.79-2.8)$ & 0.222 \\
\hline$\geq \mathrm{pT} 3 \mathrm{~b}$ & $4.59(2.57-8.2)$ & $<0.001$ & $1.16(0.56-2.37)$ & 0.691 \\
\hline \multicolumn{5}{|c|}{ Lymph node status } \\
\hline $\mathrm{pN} 0 / \mathrm{pNx}$ & 1 & & 1 & \\
\hline $\mathrm{pN} 1$ & $1.89(1.18-3.03)$ & 0.009 & $0.65(1.55-0.39)$ & 0.098 \\
\hline \multicolumn{5}{|c|}{ Gleason score } \\
\hline$<7$ & 1 & & 1 & \\
\hline 7 & $4.73(0.64-34.89)$ & 0.127 & $3.88(0.26-0.52)$ & 0.185 \\
\hline$>7$ & $17.34(2.41-125)$ & 0.005 & $8.5(0.12-1.13)$ & 0.038 \\
\hline \multicolumn{5}{|c|}{ Surgical margin status } \\
\hline Negative & 1 & & 1 & \\
\hline Positive & $2.34(1.56-3.52)$ & $<0.001$ & $1.51(0.66-0.97)$ & 0.066 \\
\hline \multicolumn{5}{|l|}{ Risk score } \\
\hline Low & 1 & & 1 & \\
\hline High & $5.9(3.88-8.97)$ & $<0.001$ & $4.04(0.25-2.44)$ & $<0.001$ \\
\hline
\end{tabular}

In our study, we calculated the immune/stromal score of each PCa sample extracted from the TCGA database by applying an ESTIMATE algorithm. The results showed that a higher immune/stromal score was associated with a poorer DFS, higher Gleason score, and higher pathological $\mathrm{T}$ stage in PCa patients. Subsequently, we divided PCa patients into high/low immune (or stromal) score groups and identified 515 cross-sectional DEGs. The GO and KEGG analysis of DEGs showed that DEGs primarily participated in the TME, such as immune responses, inflammatory responses, cell adhesion, extracellular matrix organization, and leukocyte migration. These processes may inhibit tumor progression and metastasis, thereby improving DFS. We found that these DEGs have a strong correlation with the immune response and tumor immune microenvironment. In addition, we applied univariate Cox and LASSO Cox regression models to construct a prognostic signature model based on 18 DEGs. In this model, the DFS in the high-RS group was significantly lower than that in the low-risk group, and the AUCs for the 1-year, 3-year and 5-year DFS rates were 0.890, 0.877 and 0.841 , respectively; thus, recurrence in PCa patients could be well predicted. In addition, the stratified analysis showed that the RS model also had 
strong prognostic capability for PCa patients with negative surgical margins (R0). Our model has more advantages in predicting the accuracy of prognosis than other models in other studies (the AUC of the ROC curve varied from 0.605-0.768) [22-24] and is expected to be applied to the clinical prognosis assessment of PCa patients.

Among the RS models, the expression levels of C1QC, COL1A1, HOPX, ITGAX, STAB1, and TGFB1 were low, and the prognosis was good. In contrast, we found that the low expression levels of other genes were related to the poor prognosis of PCa patients. $\mathrm{C} 1 \mathrm{QC}$ belongs to $\mathrm{C} 1 \mathrm{Q}$ and plays an important role in adaptive and innate immune responses. Studies have shown that C1QC can promote the adhesion, migration and proliferation of malignant pleural mesothelioma [25], as well as the increase in C1QC levels in patients with sarcoma, and C1Q1 is associated with poor prognosis [26]. High expression of the COL1A1 gene will cause unrestricted growth factors which, in turn, will benefit tumor proliferation [27]. The HOPX gene may be involved in the malignant transformation of cancer cells. Studies have shown that higher HOPX expression is an independent adverse prognostic factor for acute myeloid leukemia [28]. The expression level of ITGAX is positively correlated with aggressive prostate cancer [29]. STAB1 is an identified oncogene whose increased expression promotes tumorigenesis and tumor progression [30], and it is associated with poor prognosis in many cancers. TGFB1 is often up-regulated in tumor cells and highly secreted into the prostate environment, partially mediating the immunosuppressive effect on NK cells and promoting the invasion and metastasis of prostate cancer [31].

APOF can act as a tumor suppressor for hepatocellular carcinoma, and the decreased expression of APOF is associated with a poor prognosis [32]. The genetic variation in the nicotinic cholinergic receptor gene (CHRNS) may affect the risk of lung cancer [33]. The low expression of CLIC6 in breast cancer is related to a high histological grade [34]. The tumor suppressor gene EGR-1 can directly mediate the apoptotic function through the transcriptional upregulation of Bax-mRNA and protein and the increase of oligomerization and activation [35]. FEV is rich in alanine c-terminal, indicating that it may act as a transcriptional inhibitor [36]. FOS is considered a regulator of cell proliferation, differentiation, and transformation and participates in the MAPK signaling pathway $[37,38]$. GJB1 is abundantly expressed in other well-differentiated cell types such as prostate and pancreas. In prostate tumors, the ability to assemble GJS from GJB1 and GJA1 is impaired [39]. GNG2 is involved in the signal transduction of the GPCR and CCR5 pathways in macrophages, and the expression level of GNG2 in malignant melanoma is decreased $[40,41]$. The protein encoded by the HSD11b1 gene is a microsomal enzyme involved in the synthesis and regulation of prostaglandins. Up-regulation of OLFML3 enhances self-renewal of glioma stem cells and triggers primary tumor immunity, and PLTP plays a crucial role in mediating the association between triacyl lipid A and lipoprotein, which is beneficial to the anticancer properties [42]. As a candidate cancer suppressor, low TGM3 expression is associated with a poor overall survival rate in patients with neck cancer [43].

The RS prognostic model constructed by these 18 genes has not been reported and may represent a new prognostic factor for PCa. Furthermore, the multivariate Cox model showed that the RS and Gleason score were two independent prognostic indicators. To provide personalized scores for the prognosis of each $\mathrm{PCa}$ patient, a nomogram combining the TME-related RS and Gleason score for the prediction of DFS rate was established. The AUCs for the 1-year, 3-year and 5-year DFS rates of the nomogram were 0.9070 .893 and 0.872 , respectively. However, the ROC curves based on the Gleason score model only showed that the AUCs for the 1-year, 3-year and 5-year DFS rates were 0.724, 0.695 and 0.688 , respectively. The combination of the TME-related RS and Gleason score was shown to have better prognostic value than the Gleason score alone.

Finally, we used CIBERSORT and LM22 to jointly estimate the scores of 22 human immune cell types in PCa samples, and compared differences in the composition of immune cell types and the expression levels of five immune checkpoints (CTLA-4, PD-1, LAG-3, TIM-3 and TIGIT) between high-RS and low-RS groups. The concentrations of M2 macrophages and Tregs were higher in the high-RS group. In contrast, the low-RS group had a higher proportion of CD $8 \mathrm{~T}$ cells, resting memory CD $4 \mathrm{~T}$ cells, and M1 macrophages. The expressions levels of CTLA-4, PD-1, LAG-3, TIM-3 and TIGIT in high-RS patients were significantly higher than those in low-RS patients $(P<0.05)$. Previous studies have shown that resting memory CD $4 \mathrm{~T}$ cells can further differentiate and have multiple functions, including restoring immune tolerance to autoantigens or heteroantigens and promoting CD $8 \mathrm{~T}$ cells actions against tumors [44, 45]. Tregs expressing CTLA-4 play a crucial role in the maintenance of immunological self-tolerance and homeostasis and suppressing the anti-tumor immune response [46]. CTLA-4 is expressed in activated CD 4 and CD 8 T cells, which can terminate the response of activated $\mathrm{T}$ cells and 
mediate the inhibitory function of Tregs [47]. Overexpression of PD-1 on CD $8 \mathrm{~T}$ cells is an indicator of T-cell depletion [48]. Inhibiting or knocking out LAG-3 will release the inhibitory function of Tregs on $\mathrm{T}$ cells. TIM-3 suppresses anti-tumor immunity by mediating T-cell depletion. TIGIT can suppress immune cells in multiple steps of the tumor immune cycle [49]. In our study, the proportion of Tregs in high-RS patients was higher, the expression levels of the immune checkpoints CTLA-4, PD-1, LAG-3, TIM- 3 and TIGIT were higher, and the prognosis was poor, suggesting that the immunosuppressive environment and the high expression of immune checkpoints may be the reasons for the poor prognosis of PCa. In addition, these results suggested that anti-CTLA4 drugs blocking immune checkpoints leads to T-cell activation, which is an ideal strategy for treating cancer. Anti-immune checkpoint antibody treatment will be more beneficial to high-risk PCa patients than low-risk patients, resulting in a better prognosis.

However, this study also has certain limitations. First, this study only conducted bioinformatics research using public databases. Next, we should verify the results of this study using clinical patients in a study with the prospective design. Second, the 18 hub genes related to immune cell infiltration should be further studied to clarify the regulatory mechanism of PCa immune infiltration.

\section{Conclusion}

Our study established and validated a model of RS based on 18 TME-related genes, which provided a theoretical basis for predicting the DFS of PCa and further demonstrated the TME-related features associated with tumor immune cell infiltration. These genes may be of great significance for the individualization of treatment and immunotherapy for PCa patients and postoperative rehabilitation management.

\section{Abbreviations}

TME: tumor microenvironment; PCa: prostate cancer; RP: radical prostatectomy; TCGA: The Cancer Genome Atlas; GEO: Gene Expression Omnibus; ESTIMATE: Estimation of Stromal and Immune cells in Malignant Tumor tissues using Expression data; CIBERSORT: Cell type Identification by Estimating Relative Subpopulations of RNA Transcription; FPKM: fragments per kilobase million; TPM: transcripts per million; GDC: Genomic Data Commons; DEGs: differentially expressed genes; GO: Gene ontology; BPs: biological processes; MFs: molecular functions; CCs: cellular components; KEGG: Kyoto Encyclopedia of Genes and Genomes;
IHC: immunohistochemistry; DFS: disease-free survival; LASSO: least absolute shrinkage and selection operation; RS: risk score; Coeff: coefficient; ROC: receiver operating characteristic; AUC: under the curve; K-M: Kaplan-Meier; FDR: false discovery rate; HR: hazard ratio.

\section{Supplementary Material}

Supplementary figures.

http://www.jcancer.org/v12p2371s1.pdf

\section{Acknowledgements}

This study was supported by the National Key Research and Development Programme of China (Grant No's 2017YFC1307705 and 2016YFC0106907), the Science and Technology Development Programme of Henan (Grant Number: 201403007).

\section{Author Contributions}

SH Shi and H Zhao designed/planned the study. $\mathrm{H}$ Zhao and $\mathrm{XN}$ Zhang performed computational modeling, acquired and analyzed clinical data. $\mathrm{H}$ Zhao, XN Zhang, Z Shi, and BX Guo performed imaging analysis. H Zhao, XN Zhang, WL Zhang, K $\mathrm{He}$, and $\mathrm{XQ} \mathrm{Hu}$ participated in discussion of related data. H Zhao and XN Zhang drafted this manuscript. $\mathrm{SH}$ Shi reviewed and revised this manuscript. All authors have approved the final manuscript.

\section{Competing Interests}

The authors have declared that no competing interest exists.

\section{References}

1. Litwin MS, Tan HJ. The Diagnosis and Treatment of Prostate Cancer: A Review. JAMA. 2017; 317: 2532-42.

2. Bray F, Ferlay J, Soerjomataram I, Siegel RL, Torre LA, Jemal A. Global cancer statistics 2018: GLOBOCAN estimates of incidence and mortality worldwide for 36 cancers in 185 countries. CA Cancer J Clin. 2018; 68: 394-424.

3. Ye DW, Zhu Y. Prostate cancer and prostatic diseases Best of China, 2018. Prostate Cancer Prostatic Dis. 2019; 22: 1-2.

4. Bott SR. Management of recurrent disease after radical prostatectomy. Prostate Cancer Prostatic Dis. 2004; 7: 211-6.

5. Hamdy FC, Donovan JL, Lane JA, Mason M, Metcalfe C, Holding P, et al. 10-Year Outcomes after Monitoring, Surgery, or Radiotherapy for Localized Prostate Cancer. N Engl J Med. 2016; 375: 1415-24.

6. Lu X, Horner JW, Paul E, Shang X, Troncoso P, Deng P, et al. Effective combinatorial immunotherapy for castration-resistant prostate cancer. Nature. 2017; 543: 728-32.

7. Neal JT, Li X, Zhu J, Giangarra V, Grzeskowiak CL, Ju J, et al. Organoid Modeling of the Tumor Immune Microenvironment. Cell. 2018; 175: 1972-88.e16.

8. Ni J, Wu Y, Qi F, Li X, Yu S, Liu S, et al. Screening the Cancer Genome Atlas Database for Genes of Prognostic Value in Acute Myeloid Leukemia. Front Oncol. 2019; 9: 1509.

9. Gentles AJ, Newman AM, Liu CL, Bratman SV, Feng W, Kim D, et al. The prognostic landscape of genes and infiltrating immune cells across human cancers. Nat Med. 2015; 21: 938-45.

10. Cancer Genome Atlas Research N. The Molecular Taxonomy of Primary Prostate Cancer. Cell. 2015; 163: 1011-25.

11. Coordinators NR. Database resources of the National Center for Biotechnology Information. Nucleic Acids Res. 2016; 44: D7-19.

12. Yoshihara K, Shahmoradgoli M, Martinez E, Vegesna R, Kim H, Torres-Garcia $\mathrm{W}$, et al. Inferring tumour purity and stromal and immune cell admixture from expression data. Nat Commun. 2013; 4: 2612. 
13. Li J, Li X, Zhang C, Zhang C, Wang H. A signature of tumor immune microenvironment genes associated with the prognosis of nonsmall cell lung cancer. Oncol Rep. 2020; 43: 795-806.

14. Li B, Geng R, Wu Q, Yang Q, Sun S, Zhu S, et al. Alterations in Immune-Related Genes as Potential Marker of Prognosis in Breast Cancer. Front Oncol. 2020; 10: 333.

15. Luo J, Xie Y, Zheng Y, Wang C, Qi F, Hu J, et al. Comprehensive insights on pivotal prognostic signature involved in clear cell renal cell carcinoma microenvironment using the ESTIMATE algorithm. Cancer Med. 2020; 9: 4310-23.

16. Ritchie ME, Phipson $\mathrm{B}, \mathrm{Wu} \mathrm{D}, \mathrm{Hu} \mathrm{Y}$, Law $\mathrm{CW}$, Shi $\mathrm{W}$, et al. limma powers differential expression analyses for RNA-sequencing and microarray studies. Nucleic Acids Res. 2015; 43: e47.

17. Bovelstad HM, Nygard S, Storvold HL, Aldrin M, Borgan O, Frigessi A, et al. Predicting survival from microarray data--a comparative study. Bioinformatics. 2007; 23: 2080-7.

18. Chai RC, Li YM, Zhang KN, Chang YZ, Liu YQ, Zhao Z, et al. RNA processing genes characterize RNA splicing and further stratify lower-grade glioma. JCI Insight. 2019; 5: e130591.

19. Newman AM, Liu CL, Green MR, Gentles AJ, Feng W, Xu Y, et al. Robust enumeration of cell subsets from tissue expression profiles. Nat Methods. 2015; $12: 453-7$.

20. Nguyen PL, Ma J, Chavarro JE, Freedman ML, Lis R, Fedele G, et al. Fatty acid synthase polymorphisms, tumor expression, body mass index, prostate cancer risk, and survival. J Clin Oncol. 2010; 28: 3958-64.

21. Wang $\mathrm{H}, \mathrm{Wu} \mathrm{X}$, Chen Y. Stromal-Immune Score-Based Gene Signature: A Prognosis Stratification Tool in Gastric Cancer. Front Oncol. 2019; 9: 1212.

22. Strand $\mathrm{SH}$, Bavafaye-Haghighi E, Kristensen H, Rasmussen AK, Hoyer S, Borre $\mathrm{M}$, et al. A novel combined miRNA and methylation marker panel (miMe) for prediction of prostate cancer outcome after radical prostatectomy. Int J Cancer. 2019; 145: 3445-52.

23. Wang Y, Yang Z. A Gleason score-related outcome model for human prostate cancer: a comprehensive study based on weighted gene co-expression network analysis. Cancer Cell Int. 2020; 20: 159.

24. Wang Y, Lin J, Yan K, Wang J. Identification of a Robust Five-Gene Risk Model in Prostate Cancer: A Robust Likelihood-Based Survival Analysis. International journal of genomics. 2020; 2020: 1097602.

25. Zhang J, Chen $\mathrm{M}$, Zhao $\mathrm{Y}$, Xiong $\mathrm{H}$, Sneh $\mathrm{T}$, Fan $\mathrm{Y}$, et al. Complement and coagulation cascades pathway correlates with chemosensitivity and overall survival in patients with soft tissue sarcoma. Eur J Pharmacol. 2020; 879: 173121.

26. Mangogna A, Belmonte B, Agostinis C, Zacchi P, Iacopino DG, Martorana A, et al. Prognostic Implications of the Complement Protein $\mathrm{C} 1 \mathrm{q}$ in Gliomas. Front Immunol. 2019; 10: 2366.

27. Lichner Z, Ding Q, Samaan S, Saleh C, Nasser A, Al-Haddad S, et al. miRNAs dysregulated in association with Gleason grade regulate extracellular matrix, cytoskeleton and androgen receptor pathways. J Pathol. 2015; 237: 226-37.

28. Lin CC, Hsu YC, Li YH, Kuo $Y Y$, Hou HA, Lan KH, et al. Higher HOPX expression is associated with distinct clinical and biological features and predicts poor prognosis in de novo acute myeloid leukemia. Haematologica. 2017; 102: 1044-53.

29. Williams KA, Lee M, Hu Y, Andreas J, Patel SJ, Zhang S, et al. A systems genetics approach identifies CXCL14, ITGAX, and LPCAT2 as novel aggressive prostate cancer susceptibility genes. PLoS Genet. 2014; 10: e1004809.

30. Lin SY, Hu FF, Miao YR, Hu H, Lei Q, Zhang Q, et al. Identification of STAB1 in Multiple Datasets as a Prognostic Factor for Cytogenetically Normal AML: Mechanism and Drug Indications. Mol Ther Nucleic Acids. 2019; 18: 476-84.

31. Gao F, Alwhaibi A, Sabbineni H, Verma A, Eldahshan W, Somanath PR. Suppression of Akt1-beta-catenin pathway in advanced prostate cancer promotes TGFbeta1-mediated epithelial to mesenchymal transition and metastasis. Cancer Lett. 2017; 402: 177-89.

32. Wang YB, Zhou BX, Ling YB, Xiong ZY, Li RX, Zhong YS, et al. Decreased expression of ApoF associates with poor prognosis in human hepatocellular carcinoma. Gastroenterol Rep (Oxf). 2019; 7: 354-60.

33. McKay JD, Hung RJ, Han Y, Zong X, Carreras-Torres R, Christiani DC, et al. Large-scale association analysis identifies new lung cancer susceptibility loci and heterogeneity in genetic susceptibility across histological subtypes. Nat Genet. 2017; 49: 1126-32.

34. Ko JH, Ko EA, Gu W, Lim I, Bang H, Zhou T. Expression profiling of ion channel genes predicts clinical outcome in breast cancer. Mol Cancer. 2013; 12 : 106.

35. Zagurovskaya M, Shareef MM, Das A, Reeves A, Gupta S, Sudol M, et al. EGR-1 forms a complex with YAP-1 and upregulates Bax expression in irradiated prostate carcinoma cells. Oncogene. 2009; 28: 1121-31.

36. Renzi S, Anderson ND, Light N, Gupta A. Ewing-like sarcoma: An emerging family of round cell sarcomas. J Cell Physiol. 2019; 234: 7999-8007.

37. Sharma NV, Pellegrini KL, Ouellet V, Giuste FO, Ramalingam S, Watanabe K, et al. Identification of the Transcription Factor Relationships Associated with Androgen Deprivation Therapy Response and Metastatic Progression in Prostate Cancer. Cancers (Basel). 2018; 10: 379.

38. Schlomm T, Hellwinkel OJ, Buness A, Ruschhaupt M, Lubke AM, Chun FK, et al. Molecular cancer phenotype in normal prostate tissue. Eur Urol. 2009; 55: 885-90.
39. Ray A, Katoch P, Jain N, Mehta PP. Dileucine-like motifs in the C-terminal tail of connexin 32 control its endocytosis and assembly into gap junctions. J Cell Sci. 2018; 131: jcs207340.

40. Yajima I, Kumasaka MY, Yamanoshita O, Zou C, Li X, Ohgami N, et al. GNG2 inhibits invasion of human malignant melanoma cells with decreased FAK activity. Am J Cancer Res. 2014; 4: 182-8.

41. Yajima I, Kumasaka MY, Naito Y, Yoshikawa T, Takahashi H, Funasaka Y, et al. Reduced GNG2 expression levels in mouse malignant melanomas and human melanoma cell lines. Am J Cancer Res. 2012; 2: 322-9.

42. Chen P, Hsu WH, Chang A, Tan Z, Lan Z, Zhou A, et al. Circadian Regulator CLOCK Recruits Immune-Suppressive Microglia into the GBM Tumor Microenvironment. Cancer Discov. 2020; 10: 371-81.

43. Wu X, Cao W, Wang X, Zhang J, Lv Z, Qin X, et al. TGM3, a candidate tumor suppressor gene, contributes to human head and neck cancer. Mol Cancer. 2013; 12: 151.

44. Rosenberg J, Huang J. CD8(+) T Cells and NK Cells: Parallel and Complementary Soldiers of Immunotherapy. Curr Opin Chem Eng. 2018; 19: 9-20.

45. Crouse J, Xu HC, Lang PA, Oxenius A. NK cells regulating T cell responses: mechanisms and outcome. Trends Immunol. 2015; 36: 49-58.

46. Sakaguchi S, Mikami N, Wing JB, Tanaka A, Ichiyama K, Ohkura N. Regulatory T Cells and Human Disease. Annu Rev Immunol. 2020; 38: 541-66.

47. Rowshanravan B, Halliday N, Sansom DM. CTLA-4: a moving target in immunotherapy. Blood. 2018; 131: 58-67.

48. Wherry EJ, Kurachi M. Molecular and cellular insights into T cell exhaustion. Nat Rev Immunol. 2015; 15: 486-99.

49. Anderson AC, Joller N, Kuchroo VK. Lag-3, Tim-3, and TIGIT: Co-inhibitory Receptors with Specialized Functions in Immune Regulation. Immunity. 2016; 44: 989-1004. 